\section{Moda y Arte. Campos en intersección}

María Valeria Tuozzo * - Paula López ${ }^{\star *}$

\begin{abstract}
Resumen: Se investigan los vínculos entre indumentaria y arte, como campos de la práctica social, cuya intersección en los tiempos actuales -caracterizados por la preeminencia de la comunicación, la imagen, la experimentación, la estética y la singularidad como premisas- confluyen en el diseño, en general, y el diseño de autor, en particular. Se complejiza el concepto de moda masiva -en tanto cíclica, industrializada, efímera- en contraposición al arte y al diseño de indumentaria -abierto, experimental, singular-y se indaga en los conceptos de campo de la indumentaria y de los artistas. Se presenta también una línea de tiempo de los últimos cincuenta años, con un recorte de fenómenos que manifiestan estos vínculos entre indumentaria y arte, a nivel local y contemporáneo.
\end{abstract}

Palabras clave: arte aplicado - campos - contemporáneo - diseño - experimental - indumentaria - local - masivo - sociedad - vínculos.

[Resúmenes en italiano, inglés y portugués en la página 134]

(*) Licenciada en Sociología, egresada de la Facultad de Ciencias Sociales de la Universidad de Buenos Aires. Docente en la Facultad de Diseño y Comunicación de la Universidad de Palermo y en la Facultad de Diseño y Urbanismo de la UBA.

${ }^{(* *)}$ Diseñadora Gráfica, egresada de la Facultad de Arquitectura Diseño y Urbanismo de la Universidad de Buenos Aires. Se desempeña principalmente en el área editorial (coleccionables, revistas y libros) e infografía y organiza equipos de trabajo. Durante varios años, se desempeñó como docente y Jefe de Trabajos Prácticos en la Facultad de Diseño y Urbanismo de la UBA.

Dentro del vasto campo de la indumentaria, no todo es Moda. La Indumentaria, como categoría más abarcadora, incluye al fenómeno de la Moda. Es "el hecho de Indumentaria el objeto propio de la investigación sociológica o histórica” (Barthes, 2003, p. 355).

Se enmarcan también todo tipo de trajes que no dependen de la lógica de la Moda (como uniformes, los trajes regionales o ropa de trabajo), y como otro sector, dentro del amplio campo de la Indumentaria, hay experimentación y hay Arte.

Constituidos como campos, el de la Indumentaria, el de la Moda y el del Arte, tienen su lógica de funcionamiento. Siguiendo a Bourdieu, se denomina campo "a un espacio de juego, a un campo de relaciones objetivas entre los individuos o las instituciones que compiten por un 
juego idéntico" (Bourdieu en Croci y Vitale, 1990, p. 91).

El Arte se presenta como otro campo de lo social, que configura un cierto perfil vinculado con la Moda y la Indumentaria como otros campos; ya sea porque existen artistas que se dedican a producir Indumentaria o diseñadores que crean Arte en su quehacer de Diseño.

\section{El fenómeno del Siglo XX}

La Moda es el fenómeno de la masividad: algún gusto, práctica, objeto o forma de vestir que se adopta extendidamente; es efimera, transitoria, cambia con periodicidad, se nutre del consumo y se difunde por medios multitudinarios y es por esto que su presencia se ha visto acentuada con la Revolución Industrial y los medios de producción del capitalismo e incentivada a través de la cultura de masas del siglo XX. Dice Susana Saulquin que "...la forma Moda se convierte en una lógica ordenada, aunque no regular, de la distinción social, del cambio y de la construcción de identidades" (Saulquin, 2010, p. 18). Cabe aclarar que la regularidad en los ciclos de la Moda aparece mucho después de su gestación que se marca históricamente para el 1350-1370; la regularidad mencionada adviene con la formalización de los medios de producción del capitalismo, el mercado y el establecimiento de las temporadas.

La Moda es siempre un hecho de Indumentaria; su origen, sin embargo, puede representar uno u otro movimiento. La Moda puede ser tanto un hecho de Indumentaria artificialmente elaborado por especialistas (la alta costura, por ejemplo) como constituirse por la propagación de un simple hecho de vestuario, reproducido a escala colectiva por motivos diversos (Barthes, 2003, p. 356).

Aquí se entiende al hecho de indumentaria como práctica social y al vestir como una práctica individual. En este sentido, Barthes conceptualiza dichos fenómenos en analogía con la definición de Lengua y de Habla. Por lo tanto y de acuerdo a lo expuesto, la Moda es comprendida dentro del ámbito de la indumentaria con la particularidad que la representa: masividad, industrialización, cíclica; aunque por supuesto, en medio del pasaje al siglo XXI, en la actualidad dichos ciclos son cada vez más efímeros y sus características en plena transformación a la era digital.

\section{¿Es el modelo único una obra de Arte?}

La Alta Costura ha sido la del modelo único y el genio del creador. En esta concepción, la orientación del modelo hacia la obra de Arte, es donde se hace preciso señalar cuáles son las grandes y/o sutiles diferencias que distinguen a la Moda (producción en serie) del Arte (creación del modelo único) y viceversa, así como también dedicar atención a sus similitudes.

Constituidos entonces como campos, el de la Moda y el del Arte, cada uno tiene su lógica de funcionamiento. Así, por ejemplo, dentro del campo del Moda, la alta costura ilustra su juego y establece cierto tipo de relaciones; como Bourdieu define 
En este campo particular que es el mundo de la alta costura los dominantes son los que poseen en mayor grado el poder de constituir objetos como algo raro por el proceso de la firma (la "griffe"); son aquellos cuya firma tiene el precio más alto (Bourdieu en Croci y Vitale, 1990, p. 91).

En el campo del Arte, es la obra de Arte, como producción del modelo único, la que también se caracteriza en parte, con el juego de la firma, como en la alta costura. El autor es la entidad que le brinda identidad y valor a la obra, al vestido, al modelo.

Una provocativa frase de Max Ernst (artista del movimiento dadá y del surrealismo), contrasta las lógicas del Arte y de la Moda: Let there be fashion, may art perish (Que haya moda, que el arte perezca). Esta provocación de Max Ernst en1919, contrasta los valores perdurables del Arte con la superficialidad y lo efímero de la Moda (Hayward Gallery, 1999, p. 97).

Como diferencia sustancial, se puede señalar que

La distinción entre entender un objeto como 'de consumo' o 'consumible', aceptando su duración limitada como característica, y el objeto en cuestión como documento o ilustración de dicho consumo, es lo que separa a la Moda del Arte. Cuando un objeto se ha aceptado como Moda, de inmediato cesa de existir. Como postuló el sociólogo Georg Simmel al comienzo del siglo pasado, la Moda muere en el mismo momento en que empieza a existir, en la instancia en que el corte de un vestido o la forma de un abrigo se acepta en la corriente cultural principal. Para garantizar su supervivencia en la cultura de la mercancía, la Moda tiene que reinventarse constantemente a sí misma y proclamar un nuevo estilo que suplante al anterior. El Arte moderno, en cambio, empieza a existir sólo cuando sus formas progresistas son canonizadas (Steele, 2005, p. 88).

Sin embargo, la intersección de los campos del Arte y la Moda se manifiesta de diversas maneras. Cada uno de los campos se ve alimentado por lo que emana del otro: la masividad de la Moda para la obra de Arte, el aura cultural de la obra de Arte para el diseño en la Moda. Estas transferencias y espacios que comparten se manifiestan de esta forma:

La moda utiliza el arte, análogo a la historia, como un modelo visual para su interpretación contemporánea. La elevada posición que es dada a las bellas artes en la cultura occidental es empleada por la Moda para aumentar el capital cultural de sus creaciones. Cuando el diseño de moda exhibe una referencia abierta a un estilo o motivo pictórico, o cita un trabajo artístico particular, la posición y el valor que el artista o trabajo ha acumulado en el curso de la historia, son también transferidos a esos diseños. Esta transferencia ocurre de varias maneras:

1. el artista que deviene diseñador de Moda. Un ejemplo es la indumentaria de caballeros de Giacomo Balla de 1914, que cita sus propias pinturas;

2. el diseñador emplea artistas para la decoración de sus trajes, como cuando Salvador Dalí trabajó para Elsa Schiaparelli en 1937; 
3. la Moda convierte un estilo contemporáneo de pintura en diseño decorativo de un vestido, como en la colección Pop Art de 1966 de Yves Saint Laurent; 4. la presentación de una colección deviene en cuadro viviente artístico-histórico, como en la pasarela de Viviente Westwood de 1994, que citó las obras de Franz-Xaver Winterhalter y otros artistas del Segundo Imperio;

5. la interpretación de una publicación u otras formas mediáticas de promoción, que inserta a la Moda en un ambiente de Arte, como las fotos de Kart Lagerfeld de 1997, que deliberadamente copió motivos de la Bauhaus (Steele, 2005, p. 90).

A pesar de que alguna concepción del Arte, descarta la posibilidad de empleo o utilidad de la obra de Arte, que quedaría en ese caso por fuera de la conceptualización del Arte, con una actitud contemplativa, postula que "el trabajo del artista es mirado como no instrumental -no se puede hacer nada con una pintura excepto mirarla-"(Hayward Gallery, 1999, p. 7), en el campo de la Indumentaria, otros referentes consideran a la creación en lo que vestimos como Arte aplicado: "La Moda es Arte aplicado", dice Felisa Pinto (La Nación, 2012), o como consigna la artista Dalila Puzzovio en su sitio web, es "Un acto feliz", y se explaya: "Los artistas, para lograr ese cometido celestial de la creación, deben ser curiosos, perceptivos, enérgicos, carismáticos, inseguros, ingenuos, narcisistas y, sobre todo, muy disciplinados. La Moda es un acto feliz muy serio. Debe estar lleno de sonrisas serias."

\section{Algunos hitos en Arte e Indumentaria}

En el ámbito local y contemporáneo, algunos hitos que pueden citarse, pueden verse en línea de tiempo en la imagen (Ver Figura1, pg 127), como expresiones de experimentación y vínculos entre Arte y Moda y considerando arte contemporáneo al arte que produce en el punto presente del tiempo o el arte que se produjo desde la Segunda Guerra Mundial (los museos del arte contemporáneo definen comúnmente sus colecciones como el arte producido desde ese punto histórico). Como artista que deviene diseñador de Moda podríamos mencionar a Dalila Puzzovio con sus Dobles Plataformas, de 1967 Premio Internacional Instituto di Tella, muy documentado en su sito web www.dalilapuzzovio.com.ar/; y tiempo después, su presentación El Deslumbre (obra y performance con zapatos) en Espacio Patio Bullrich, arteBA 2011, cuyo registro puede verse en YouTube, http://youtu.be/j4HuctNtnjs. También podemos mencionar a Mary Tapia, que comenzó con su Exhibición Pachamama prêt-à-porter, en Instituto DiTella de 1969, y en 2007 tuvo su exhibición retrospectiva en Identidad criolla, en MALBA, una muestra centrada en la vuelta a la moda folk, curada por Felisa Pinto y Victoria Lescano, que incluyó la Retrospectiva Mary Tapia (Colecciones privadas 1966-2006) y con destacados diseñadores locales.

Emblemáticos y recordados, los muy experimentales en la Bienal de Arte Joven de 1988, Centro Cultural Recoleta/ Mónica Van Asperen, Andrés Baño, Gabriel Grippo, Gaby Bunader y otros y más adelante, el Desfile Al borde del siglo Fundación Banco Patricios/ Gabriel Grippo, Gaby Bunader, Cristian Delgado, Andres Baño y Sergio de Loof y Kelo Romero, así como el Desfile 90/60/90 Fundación Banco Patricios/ Andres Baño y Sergio de Loof, como presentaciones de una colección que deviene en cuadro viviente artístico-histórico en sí mismo. 


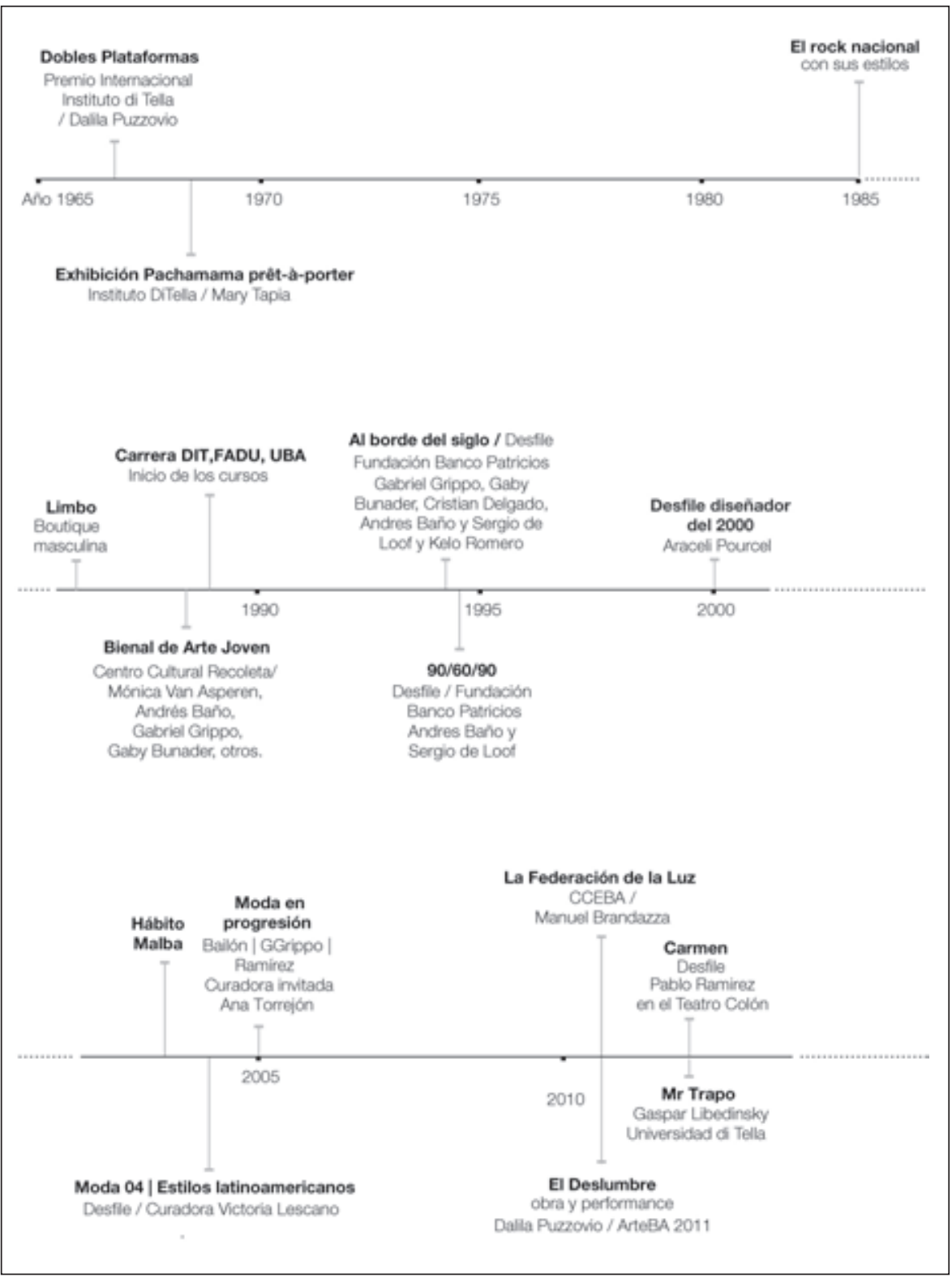

Figura 1. Algunos hitos en Arte e Indumentaria. Fuente. Tuozzo - López. 
Por otra parte, la Moda (con su necesidad constante de reconversión y en especial en la actualidad, comenzando a recorrer el siglo XXI) y las marcas comerciales dentro del campo de la Moda, no son ajenas a estos nuevos perfiles e identidades en formación. Con consecuencias simbólicas y concretas se manifiestan en el mercado: muchas son las marcas comerciales que establecen relaciones con diseñadores con su firma y su estilo para poder singularizarse. Es el caso de Muaa y Mariano Toledo, el de Ricky Sarkany con siete artistas para "Sarkany Heart" y el caso de algunos emprendimientos de la marca Ver, entre muchísimas otras acciones de marcas comerciales vinculándose con el Arte.

\section{La Moda visita las galerías y los museos}

En tanto el museo y las galerías son los lugares específicos por donde circula el Arte, la Moda ha sido y es de las calles; idea que se refuerza con la creciente cantidad de espacios en la red, como blogs, páginas, redes sociales donde se retratan las Modas y sus portadores.

Sin embargo, se puede consignar una confluencia de estos campos con mucha actualidad: las exhibiciones de Indumentaria y Moda en museos y galerías, ámbitos que fueron tradicionalmente reservados para el Arte. Un aspecto de este cruce es que

El Arte contemporáneo cita a la Moda no sólo como modelo estético sino también como campo de referencia en donde los desafíos y peligros de la vida moderna son glamorosamente puestos fuera de juego. El compromiso de la Moda con el Arte contemporáneo es también curatorial, esto es, en exhibir -a menudo experimentalmente- indumentaria en museos y galerías, equiparando Indumentaria y Arte en exhibiciones sobre objetos materiales o nociones de belleza, o usando a la industria de la Moda para financiar proyectos artísticos. La curatoria de Moda conduce en algunos casos al apoyo institucional de colecciones, por ejemplo, el primer desfile del dúo holandés Víctor and Rolf fue posible sólo a través del apoyo y políticas de adquisición del Central Museum de Utrecht y el Groninger Museum. Esto implica el posicionamiento de la Moda en la cultura contemporánea como una entre muchas manifestaciones intercambiables, más que un medio estructuralmente distinto dentro de una jerarquía cultural. El uso de los materiales básicos de la Moda (textiles, telas) y significativamente, sus modos de representación a través de fotografías, desfiles, y más, es usado en el Arte contemporáneo para acompañar la puesta en escena de la industria cultural en la modernidad tardía (Steele, 2005, p. 91).

Localmente, en Buenos Aires, podemos recordar las exhibiciones Hábito Malba, realizado en diciembre de 2003, y Moda 04 I Estilos latinoamericanos, desfiles organizados por la curadora Victoria Lescano, convocó al diseñador brasileño Alexandre Herchcovitch y a los argentinos Romero y Araceli Pourcel para desarrollar el programa de moda en el museo. También se presentó Moda en progresión, curada por Ana Torrejón, con las creaciones de Rosa Bailón, Gabriel Grippo y Pablo Ramírez, en 2005, continuando ese ciclo. 
Más actualmente, la Moda que ha visitado las galerías y los museos, también lo ha hecho con propuestas muy personales y singulares de experimentación de Indumentaria mixturados con el Arte. Por ejemplo cuando un diseñador ha creado Indumentaria a través de procesos experimentales, innovando en las formas o en los materiales (podemos citar la exhibición Mr. Trapo, de Gaspar Libedinsky, 2012 en la Universidad di Tella), o cuando un artista ha tomado formas o siluetas de la Indumentaria para hacer una creación estética; podemos citar las exhibiciones más actuales de la artista Mónica Van Asperen y que, por ejemplo, algunas de sus obras se encuentran en la colección de arte argentino contemporáneo del Museo de Arte Contemporáneo de Rosario (www.macromuseo.org.ar/coleccion/artista/v/van_asperen_monica.html).

También pueden citarse eventos que, con la plataforma de una institución, ha salido a espacios urbanos, como la performance y desfile La Federación de la Luz por Manuel Brandazza -creador argentino de indumentaria-, en 2011 -que recorrió en el centro de la ciudad de Bs. As., desde CCEBA en la calle Florida hasta Plaza San Martín- (www.wipe.com.ar/otros/750/ Brandazza---La-Federacion-de-la-luz).

\section{Una intersección: el campo del diseño}

A partir de la década de 1960, años donde comenzaron movimientos sociales de cuestionamiento de los órdenes dados, de mayor experimentación y en camino hacia la singularización de las identidades el cruce entre la Moda y el Arte en Argentina, que fue puesto en stand by por los procesos de gobiernos de facto, volvió a resurgir con un impulso innovador y creativo a fines de la década de 1980, con la Bienal de Arte Joven, donde el vestir formó parte, a la par de otras disciplinas, del Arte. En la intersección de estos campos, Indumentaria y Arte, ocurre el Diseño.

Como consecuencia de este movimiento, se inicia en 1989 la carrera de Diseño de Indumentaria y Textil en la Universidad de Buenos Aires; desde allí, la reflexión sobre la Indumentaria y la formación en Diseño se han visto fortalecidas y en pleno crecimiento.

"Con respecto al cruce entre el Arte y la Moda que se encuentra ciertamente en el diseño," dice Susana Saulquin (Saulquin, 2012). El diseño de Indumentaria supone una instancia de conceptualización de la producción del vestir que, de alguna manera tomando recursos y reflexiones del Arte, acude a medios de producción artesanal, singular e individual; con ciertos niveles de experimentación en materiales y formas. Y el Diseño se vincula también ciertamente, con la Moda, y la lógica de la serie y la producción.

En la actualidad, recorrida por lo digital, en plena transformación de las lógicas de la Indumentaria y la Moda, dice Susana Saulquin:

El creciente y previsible reemplazo de la sociedad industrial por una sociedad tecnológicamente dirigida, con redes informáticas que comunican al instante todas las zonas del planeta, condiciona a la vestimenta que, personalizada e individualista, evoluciona adaptada a nuevos parámetros. El nuevo diseño deberá responder a requerimientos que sumen la funcionalidad a la practicidad, el cuerpo real y a los nuevos materiales, y a contemplar la relación entre la naturaleza ambiental y humana (Saulquin, 2010, p. 20). 
Esta intersección entre las formas de la Indumentaria y la Moda, por un lado, y el enorme campo del Arte por otro, pueden explicarse en la actualidad con lo que García Canclini ha denominado como procesos de hibridación en términos culturales. Según este autor, estos son "procesos socioculturales en los que las estructuras o prácticas discretas, que existían en forma separada, se combinan para generar nuevas estructuras, objetos y prácticas" (García Canclini, 1999, p. 14).

Como ejemplo, con estos conceptos, se puede interpretar una colección singular como la de 1969, Exhibición Pachamama prêt-à-porter, en Instituto DiTella, por Mary Tapia. El prêt-àporter, surgido a mediado de los años cuarenta, copiaba en líneas más simples las creaciones inalcanzables de la alta costura para el público en general. En la conceptualización de Mary Tapia, se cruzan la simplificación de la producción industrial y la posibilidad de seriar esa producción; por otra parte, los materiales y la referencia a través del nombre (de un simbólico y mítico personaje, como la Pachamama) a la cultura autóctona.

Más adelante detalla:

¿Cómo fusiona la hibridación estructuras o prácticas sociales discretas para generar nuevas estructuras y nuevas prácticas? A veces esto ocurre de modo no planeado, o es el resultado imprevisto de procesos migratorios, turísticos y de intercambio económico y comunicacional. Pero a menudo la hibridación surge de la creatividad individual y colectiva. No solo en las Artes, sino en la vida cotidiana y en el desarrollo tecnológico. Se busca reconvertir un patrimonio (una fábrica, una capacitación profesional, un conjunto de saberes y técnicas) para reinsertarlo en nuevas condiciones de producción y mercado. Aclaremos el significado cultural de reconversión: se utiliza este término para explicar las estrategias mediante las cuales un pintor se convierte en diseñador... (Bourdieu) (Canclini, 1999, pp. 16-17).

Los campos en intersección se manifiestan enriquecidos por la hibridación. La reconversión en las identidades del diseñador y el artista son de alguna manera, caras de una misma moneda, haciendo, en muchos casos, que la posible caracterización de sus labores en uno u otro ámbito sean indisolubles e imposibles de clasificar. También, generando a su vez nuevas formas de producción y circulación, así como nuevos consumidores conscientes de sus gustos y de los procesos, por los cuales sus productos de consumo son elegidos, adquiriendo así la obra misma y su creador otra entidad dentro de los campos.

\section{Lo que se llama diseño de autor}

Todo esto es lo que hace posible pensar lo que se da en llamar diseño de autor, denominación que (muy utilizada localmente en la actualidad) pone en evidencia la importancia de la firma, del autor; en tanto ese diseño tiene una particular marca que lo diferencia a través de las distintas colecciones que presenta el diseñador y que es lo que le da finalmente su personalidad. Así como sucede con la firma en la obra de Arte; señalado en líneas anteriores. 
Como caso de diseño de autor, podemos citar al diseñador Pablo Ramírez: sus colecciones tienen esa marca personal que se ve reflejada en cada presentación. En este caso, el vínculo con el Arte no está sólo en el diseño en sí, sino también, en la jerarquía de los lugares de presentación de su trabajo: su colección invierno 2012, llamada Carmen, se presentó con un desfile en el famoso Teatro Colón de la ciudad de Buenos Aires, un espacio habitualmente reservado a la alta cultura y las Artes, entre las que la Moda estaría fuera. Puede verse un registro en video en www.livestream.com/pasarelaba y en vimeo.com/38593303.

En el diseño de autor, la producción se aleja de la serie que emerge de la Moda, en los términos en que la define Baudrillard en El Sistema de los Objetos:

... el objeto no debe escapar a lo efímero y a la Moda. Esta es la característica fundamental de la serie: el objeto está sometido a una fragilidad organizada. En un mundo de abundancia (relativa) es la fragilidad la que sucede a la escasez o rareza como dimensión de lo que falta. Se mantiene a la serie, por la fuerza, en una sincronía breve, en un universo perecedero. El objeto no debe escapar a la muerte (Baudrillard, 2004, p. 165).

Así es como funciona la producción en serie que constituye a la Moda, con su objeto que caduca en el momento de la masificación, y ese objeto tiene las características de la producción seriada:

paralelamente, cuando se pasa del modelo a la serie, las cualidades sensibles del objeto disminuyen al mismo tiempo que las cualidades técnicas.(...) el vestido de lana pura o de seda natural se multiplicará en la confección en lana mezclada o en rayón. Con la materia, es el peso, la resistencia, el grano, el “calor" lo que al desaparecer, en proporciones variables, señalará la diferencia (Baudrillard, 2004, p. 166).

En cambio, en el modelo, el del diseño de autor o el modelo de la obra de arte, también: “... tiene una armonía, una unidad, una homogeneidad, una coherencia de espacio, de forma, de sustancia, de función, es una sintaxis. El objeto de serie no es más que yuxtaposición, o combinación fortuita, discurso inarticulado. Destotalizado..."(Baudrillard, 2004, p. 167).

Esta coherencia o conjunto de relaciones necesarias se destruye en provecho del juego diferencial de las formas, de los colores o de los accesorios. Al estilo le sucede una combinatoria, la descalificación que señalamos en el plano técnico cobra aquí el aspecto de una desestructuración. En el objeto modelo no hay ni detalles ni juego de detalles: los Rolls-Royce son negros y nada más que negros (Baudrillard, 2004, p. 168).

Con síntesis, los diseños de Pablo Ramírez, son negros y nada más que negros (en un guiño de coincidencia con los Rolls-Royce); y este sello es lo que le da su particular personalidad y los constituyen en modelos. Como en otros casos de diseño de autor, cuya firma además los consolida, es siempre uno y el mismo con los matices que el autor, diseñador o artista, pretende otorgarle. 
Y es que el matiz (en la unidad) corresponde al modelo mientras que la diferencia (en al uniformidad) corresponde a la serie.(...) ...si el matiz parece raro y la diferencia marginal innumerable es porque se benefician de una difusión en masa; estructuralmente, es el matiz el que es inagotable (el modelo se orienta aquí hacia la obra de Arte), la diferencia serial, por su parte, entra en una combinatoria finita, en una tablatura que cambia sin duda continuamente con la Moda, pero que, para cada momento sincrónico en que se la considera, es limitada y está estrechamente ligada a la dictadura de la producción (Baudrillard, 2004, p. 169).

\section{Consideraciones finales}

La reconversión en estos campos, el del Arte y el de la Indumentaria, genera nuevas prácticas, nuevos perfiles e identidades en formación. Nuevos y renovados capitales simbólicos enriquecen a ambos campos y los intersectan en múltiples acciones, no sin entrar en conflictos y resistencias; por ejemplo, al momento de denominar la práctica y encuadrarla dentro de uno u otro campo.

Este proceso dentro de estos campos es posible dada la mayor complejidad de lo social, al denominado proceso de globalización que trae aparejado, si bien acciones que encuadran al planeta, también una creciente localización y atomización de las acciones posibles de leerse como formas de resistencia y conservación.

La hibridación incluye producciones de formas artesanales, experimentales, singulares. Lo global y lo local, lo Artesanal y lo industrial, lo culto y lo popular por nombrar algunos binomios que se enfrentan en estos campos se ponen en juego en las luchas simbólicas de las que habla Bourdieu en búsqueda de legitimación, dentro del campo del Arte y el de la Indumentaria en esos espacios de intersección donde se experimenta y se juega con estas prácticas.

También es posible leer esta intersección de los campos y sus prácticas como una intersección entre la modernidad y la posmodernidad. La modernidad, con sus grandes relatos y campos estancos, cuyas verdades y saberes eran únicos y últimos; y luego la posmodernidad, como "copresencia tumultosa de todos (se agrega todos los estilos o prácticas), el lugar donde los capítulos de la historia del Arte y del folclore se cruzan entre sí y con las nuevas tecnologías culturales." (García Canclini, 1999, p. 308). Por supuesto, la Indumentaria queda incluida en este proceso. También como intersección de estos campos, la personalización y la individualización son tendencias, parafraseando a Susana Saulquin, los lazos en estos campos se estrechan aún más a partir de la personalización y la individualización que proponen los fines de los 90 , proceso que como se ha señalado es iniciado a partir de los 60; en la personalización, el diseño y el arte se emparentan; es la mirada del diseñador y la mirada del artista las que se ponen en juego, las que se plasman y manifiestan sobre la tela, el lienzo o cualquiera sea el material por trabajar; pero no sólo la personalización de la obra sino también la personalización que los sujetos como por- 
tadores de identidades cada vez más individuales, más fragmentadas por gustos e inclinaciones que en muchos casos resultan incompatibles constituyen una nueva subjetividad más alerta y atenta a sus prácticas de consumo. (Saulquin, 2006, p. 273).

El proceso de producción artística enfrenta al artista y su obra, experimental, singular (si bien el Arte circula por el mercado); el de la Moda es un proceso que se ajusta a los modos de producción del capitalismo y del consumo masivo: talleres, mano de obra, materias primas, mercancía. Sin embargo cada vez más uno y otro campo es permeable a lo que sucede dentro de los límites del otro. El constante diálogo ya entablado desde la Moda y la Indumentaria y el Arte; hace pensar que se ha comenzado a transitar un camino en el cual cada manifestación de estas prácticas se verá enriquecida por la otra y en consecuencia, nuevos y renovados saberes y acciones esperan en el futuro. De ello, emana la posibilidad de construir nuevas subjetividades profesionales, acordes a un nuevo mundo donde las miradas que aportan diseñadores y artistas seguirán reflejando los cimientos de la complejidad de lo social y su historia.

Continuar reflexionando acerca de las diferencias y similitudes que plantean estas prácticas desde la durabilidad de la creación artística frente a lo efímero de la Moda a las transferencias que se manifiestan, los procesos de creación y los espacios de exposición son tareas para poder así generar más conocimiento sobre el hombre y su subjetividad.

\section{Referencia Bibliográficas}

Barthes, R. (2003). El sistema de la moda y otros escritos. Buenos Aires: Paidós.

Baudrillard, J. (2004). El sistema de los objetos. Buenos Aires: Siglo XXI editores.

Bourdieu, P. (1990). Alta cultura y alta costura en Sociología y Cultura. México: Grijalbo; en Croci, P. y Vitale, A. (comp.) Los Cuerpos Dóciles. Hacia un tratado sobre la Moda. Buenos Aires: Editorial La Marca.

Catálogo. (1999). Exhibición Adressing the century: 100 years of Art and Fashion, London: Hayward Gallery.

García Canclini, N.(1999). Culturas Híbridas. México: Grijalbo.

Guerreiro, L. (2012). Retrato de una Dama, La Nación, 13 de febrero 2012.

Saulquin, S. (2011). Historia de la Moda Argentina. Argentina: Emecé.

(2010). La muerte de la moda, el día después. Buenos Aires: Paidós.

Steele, V. (editor). (2005). Encyclopedia of Clothing and Fashion, Thompson Gale, vol I.; USA.

\section{Recursos Electrónicos}

Brandazza M. Consultar www.wipe.com.ar/otros/750/Brandazza---La-Federacion-de-la-luz.

Puzzovio, D. Consultar www.dalilapuzzovio.com.ar/

Ramírez, P. Consultar www.pabloramirez.com.ar, www.livestream.com/pasarelaba y en vimeo. com/38593303.

Van Asperen, M. Consultar www.macromuseo.org.ar/coleccion/artista/v/van_asperen_monica.html 


\begin{abstract}
Si tenta di identificare i legami tra l'abbigliamento e l'arte in quanto entrambi espressione della pratica sociale, e che al momento attuale -caratterizzato dall' importanza della comunicazione, delle immagini, della sperimentazione, dell'estetica e dell' unicità come premesse- si intersecano e convergono nel disegn, in generale, ed nel disegn d'autore in particolare. Si analizza il concetto di moda di massa che è una forma ciclica, industrializzata, effimera, contrapposta all'arte ed al fashion design che è invece aperto, sperimentale, singolare, e si definiscono gli ambiti concettuali propri dell'abbigliamento e dell'arte. Si traccia inoltre la cronologia di una serie di eventi degli ultimi cinquant'anni che evidenziano i legami tra abbligliamento ed arte, a livello locale e contemporáneo.
\end{abstract}

Parole chiave: abbigliamento - arte applicato - campi - contemporaneo - disegn - esperimentale - legami - locale - massivo - società.

Summary: In this paper, we investigate the links between clothing and art, as fields of social practice, and how their intersection at the present time, converges in general design, and particularly in author design. In addition, the concept of mass fashion as cyclical, industrial and ephemeral is analyzed as opposed to art and fashion design -open, experimental and singular. The article explores also the concepts of clothing field and artists fields. It also presents a timeline of the last fifty years, with a cut of phenomena that show any link between clothing and art, local and contemporary.

Keywords: applied art - clothing - contemporary - design - experimental - fields - links - local - massive - society.

Resumo: Se pesquisam os vínculos entre indumentária e arte, como campos da prática social, cuja interseção nos tempos atuais -caracterizados por a preeminência da comunicação, a imagem, a experimentação, a estética e a singularidade como premissas- confluem no design, em geral, e o design de autor em particular. Complica-se o conceito de moda massiva -em tanto cíclica, industrializada, efêmera- em contraposição à arte e ao design de indumentária -aberto, experimental, singular- e se indaga nos conceitos de campo da indumentária e dos artistas. Apresenta-se também uma linha do tempo dos últimos cinqüenta anos, com um recorte dos fenômenos que manifestam estes vínculos entre indumentária e arte, ao nível local e contemporâneo.

Palavras chave: arte aplicada - campos - contemporâneo - design - experimental - indumentária - local - massivo - sociedade - vínculos. 